\title{
Hinokiflavone from Juniperus as Therapeutic Lead against Osteoarthritis by Inhibiting ADAMTS-5
}

\author{
Rathi Suganya P., Sukesh Kalva, Lilly M. Saleena
}

\begin{abstract}
ADAMTS-5 is an important aggrecanase that cleaves at key sites in the aggrecan core protein, in healthy and diseased cartilage. ADAMTS-5 deficient mice are protected from cartilage erosion in models of experimental arthritis. Therefore inhibition of ADAMTS-5 will be a potential cure for arthritis. In this study pharmacophore model was developed by downloading 50 ligands with IC50 value from BindingDB database. Pharmacophore Alignment and Scoring Engine (PHASE) software was used to develop ligand-based pharmacophore model for ADAMTS-5 using those 50 ligands. pIC50 ranged from 7.3149 to 5.018, of which pIC50 above 6.5 were considered as active and below 5.5 were considered as inactive. Three maximum hypotheses AAHRR, AARRR, AHRRR were generated. Pharmacophoric hypothesis AARRR.4144 had the best survival score of 3. 3D-QSAR was built for the best hypothesis with training set as $70 \%$ and atom based model was generated by keeping $1 \AA$ grid spacing and 6 as maximum number of PLS factors. Results show that AARRR.4144 has the best regression coefficient of 0.9832 and Pearson-R as 0.756. A docking study revealed the binding orientations of these inhibitors at active site amino acid residue His 373 of ADAMTS-5. The results of ligand-based pharmacophore hypothesis and atom based 3D-QSAR gave detailed structural insights as well as highlighted important binding features to design a novel therapeutically active compound against ADAMTS-5. These features where used to screen natural compounds from Dukes Database and Hinokiflavone was identified as the best inhibitor with a glide score of $-8.47 \mathrm{Kcal} / \mathrm{mol}$ against Adamts-5. Therefore further studies can be carried on this natural compound to prove as a promising drug for osteoarthritis.
\end{abstract}

Index Terms-3D-QSAR, ADAMTS-5, Aggrecan, Docking, pharmacophore.

\section{INTRODUCTION}

ADAMS (A Disintegrin And Metalloproteinase) is a peptidase protein which contains a unique integrin receptorbinding disintegrin domain, comes under the family of Metzincins. ADAMS are classified as Sheddases because they cut off or shed extracellular portions of transmembrane proteins. Two subfamilies are Snake venom metalloproteases (SVMPs) and ADAMTS (A disintegrin and metalloproteinase with thrombospondin motifs) [2]. ADAMTS-4 and ADAMTS-5 are the major aggrecanase in

Manuscript received March 26, 2011. This work was supported by GVK Biosciences.

Rathi Suganya is with SRM University, Kattankulatur, Tamil Nadu, India. (phone: 9840796846; e-mail: rathisuganya@ ktr.srmuniv.ac.in).

Sukesh Kalva is with SRM University, Kattankulatur, Tamil Nadu, India. (phone: 9941452601; e-mail: Sukesh.kalva@ ktr.srmuniv.ac.in).

Lilly M. Saleena is with SRM University, Kattankulatur, Tamil Nadu, India. (corresponding author, phone: 9840506562; e-mail: 1msaleena@ yahoo.com). human cartilage. ADAMTS-5 is synthesized in the rough endoplasmic reticulum, matures in the golgi compartment, constitutively expressed in human chondrocytes and synovial fibroblasts. ADAMTS-5 is an important aggrecanase that cleaves at key sites in the aggrecan core protein, in healthy and diseased cartilage. ADAMTS-5 deficient mice are protected from cartilage erosion in models of experimental arthritis [3]. Therefore inhibition of the ADAMTS-5 will be a potential cure for arthritis. Therapeutic effects of commercially available drugs last only for a short time due to their unfavourable pharmacokinetic profiles; therefore small target specific inhibitors could have enormous potential as new therapeutics. The overall fold of the catalytic domain resembles other metalloproteinases (MMP), but the shape of the substrate-binding site is unique. This unique binding site suggests that ADAMTS-5 recognizes different substrate motifs than MMP, ADAM and other ADAMTS enzymes. The unique binding site also increases the likely success of developing inhibitors that are specific for ADAMTS-5 [3].

Ligand-based drug designing approaches like pharmacophore mapping and quantitative structure-activity relationship (QSAR) are used in drug discovery. Database search studies for new hits and to identify important structural features for functional activity will help in identifying therapeutically stable drug without any sideeffects [11].

In a rational drug design approach, identification of the pharmacophore is the most important step in achieving the stipulated goal. Pharmacophore Alignment and Scoring Engine (PHASE) software was used to develop ligandbased pharmacophore model for ADAMTS-5. PHASE uses conformational sampling and different scoring techniques to identify common pharmacophore hypothesis, each hypothesis is accompanied by a set of aligned conformations which are necessary for the ligand to bind to the receptor [9] [10]. The developed model has the ability to find potential ADAMTS-5 inhibitors from 3D-virtual databases of drug-like molecules. The conformations of active compounds obtained from the alignment of pharmacophoric points are used to derive 3D-QSAR models. Further, the binding mode of the active molecule with the active site amino acid residues was performed by XP docking using Glide.

\section{MATERIALS AND MEthodS}

\section{A. Data set:}

For designing of novel potential ADAMTS-5 inhibitors, we downloaded 71 inhibitors available for ADAMTS-5 from the Binding $D B$ database [3]. 55 compounds from the 
output had known IC-50 values. To avoid redundancy of information, the data set was further refined by removing compounds with similar biological activity and chemical structures by CANVAS (Schrodinger, LLC, New York, US) to identify diverse compounds, out of which we selected 50 . The 50 compounds selected had IC50 values of different range therefore the values (in moles/litre) were converted into negative logarithm of IC50 (pIC50). pIC50 ranged from 7.3149 to 5.018 , of which pIC50 above 6.5 were considered as active and below 5.5 were considered as inactive and rests were moderately active.

\section{B. Ligand Preparation:}

These ligands were geometrically refined (cleaned) and conformers were generated with maximum number of conformers per structure as 1000 with force field OPLS2005 with RMSD $1.0 \mathrm{~A}^{\circ}$.

\section{Hypothesis generation:}

PHASE provides a standard set of six pharmacophore features, hydrogen bond acceptor (A), hydrogen bond donor (D), hydrophobic group $(\mathrm{H})$, negatively ionizable $(\mathrm{N})$, positively ionizable $(\mathrm{P})$, and aromatic ring (R). Common pharmacophoric sites where selected from a set of variants and with the option Create Sites, number of acceptors were modified to 2 , negatively ionizable to 0 , others were kept default. This gave 6 different variant lists AAHHR, AAHRR, AARRR, AHHRR, AHRRR and HHRRR.

Hypothesis generation was done by Find option in find Pharmacophore model, which generated three maximum hypotheses with AAHRR, AARRR, AHRRR. For these hypothesis scores were calculated for both actives and inactives by score hypothesis using an overall maximum root mean square deviation (RMSD) value of $1.2 \AA$. The quality of alignment was measured by survival score.

\section{3D-QSAR:}

Phase provides the option of doing QSAR with the selected pharmacophore hypothesis. In the alignment option, align non-model ligands were chosen so that the ligands that are not part of the active set were also included. In Build QSAR option random training set was kept as $70 \%$ and atom based model was generated by keeping $1 \AA$ grid spacing and 6 as maximum number of PLS factors.

\section{E. Finding Matches to the hypothesis}

Hypothesis derived form the pharmacophore was then further used to screen the natural compounds from duke's database[16], which has compounds with diverse activity.

\section{RESUlTS AND DisCUSSION}

ADAMTS-5 inhibitors can stop the expression of ADAMTS-5 thereby acting as a potential therapeutic drug for arthritis. In ligand based pharmacophore model we have developed a model which screened important pharmacophoric features necessary for these ligands to function as inhibitors. Training set consisted of 35 compounds, where 8 of them were active and 6 were inactive. Test set had 15 compounds. The pharmacophoric features selected for creating sites were hydrogen bond acceptor (A) and aromatic ring (R). Pharmacophore models containing three to five features were generated. The three and four featured pharmacophore hypotheses were rejected due to low value of survival score, as they were unable to define the complete binding space of the selected molecules. Five featured pharmacophore hypotheses was selected and subjected to stringent scoring function analysis.

102 different hypotheses were generated with AARRR, AHRRR and AAHRR; best 5 were shown in the Table I. Pharmacophoric hypothesis AARRR.4144 had the best survival score of 3.821. The pharmacophoric hypothesis of AARRR.4144 is shown in Fig 1. The features represented in this hypothesis are two hydrogen acceptor and three aromatic rings. The distance and angles between the different sites are presented in the Table IV and $\mathrm{V}$ respectively. QSAR results also shows that the AARRR.4144 has the best regression coefficient of 0.9832 , Pearson-R as 0.756. Result of atom-based 3D-QSAR with PLS 6 of AARRR.4144 hypothesis is shown in Table II.

The fitness score is checked for the pharmacophore model AARRR.4144. The best fitness score of 3 was with ligand number 10 (Fig: 5). Best five fitness score compounds are shown in the Table III. Scatter plots for the predicted and experimental pIC50 values for the ADAMTS5 QSAR model applied to the training set and the test set are shown in Fig 3 and 4 respectively.

\section{A. 3D-QSAR Analysis}

Inhibitory activity of the compound suggested by pharmacophore can be visualized by doing QSAR model. The results can be further used in designing novel ligands with the features derived from the pharmacophore model. The 3D-QSAR model was applied to the most active compound: 10 and the least active compound: 20 , which are shown in the Fig 6 and 7 respectively. These figures compare the most significant favourable (blue cubes) and unfavorable (red cubes) regions for the activity of the compound.

\section{B. Docking Analysis:}

Extra precision glide docking (Glide XP) was performed for the best active compound 10 and ADAMTS-5(2RJQ). The docking results show interaction between compound 10 and ADAMTS-5 in the active site region with HIS373 with a G-Score of $-9.14 \mathrm{Kcal} / \mathrm{mol}$ (Fig: 8 ). This complies with the 3D-QSAR model developed were the interaction is seen in the favourable region.

\section{Virtual Screening with Natural Compounds}

Ten natural compounds with similar pharmacophore been resulted. Virtual screening was performed for the above compounds and the best compound with good glide score $8.47 \mathrm{Kcal} / \mathrm{mol}$ was reported. (Fig 9). The natural compound is Hinokiflavone (Fig 10) which is reported as anticancer and antiviral drug in Duke's database. This is available in Araucaria bidwillii (Leaf), Juniperus communis(Leaf), Juniperus macropoda(Plant) which can be checked further for anti inflammatory also.

\section{CONCLUSIONS}

In conclusion, developing a pharmacophore model will help in identifying therapeutically potential compounds 
without any side effects. Various pharmacophoric models were developed for ADAMTS-5 using 50 ligands downloaded from Binding $D B$ database. Best hypothesis obtained was AARRR.4144 with two hydrogen bond acceptor and three aromatic rings. Compound 10 (sulfonylamino-alkanecarboxylate, 38) had the best result for which a highly predictive atom based 3D-QSAR model was generated. Atom based 3D-QSAR and docking study helps in understanding the relationship between structure and activity. The generated Pharmacophore was screened against the dukes database for natural compounds to identify the activity against ADAMTS-5.

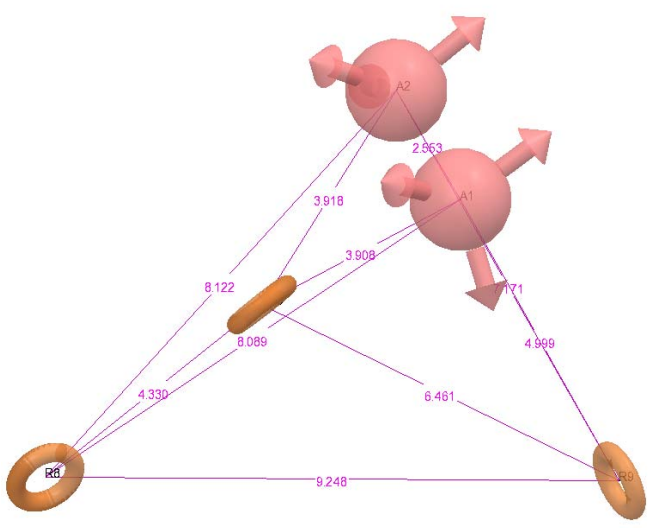

Fig 1: PHASE generated pharmacophore model AARRR.4144 illustrating hydrogen bond acceptor (A1, A2; pink), and aromatic ring (R8, R9, R10; orange) features with distances (in $\AA$ ) between different sites.

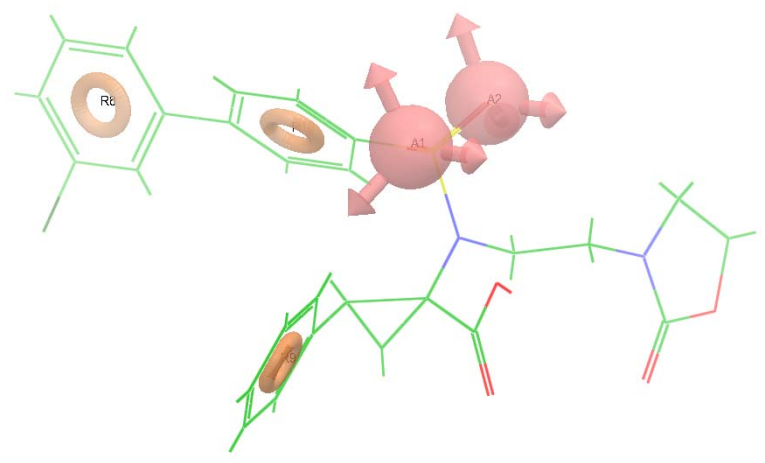

Fig 2: Best pharmacophore model AARRR.4144 aligned with molecule 10 illustrating hydrogen bond acceptor (A1, A2; pink), and aromatic ring (R8, R9, R10; orange)

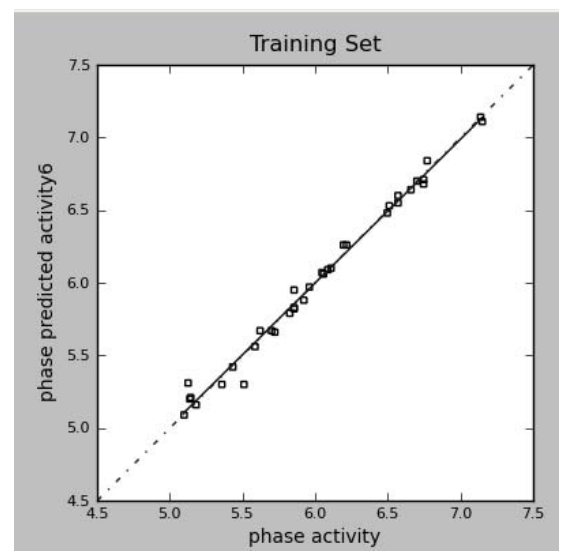

Fig 3: Scatter plots for the predicted and experimental pIC50 values for the ADAMTS-5 QSAR model applied to the training set.

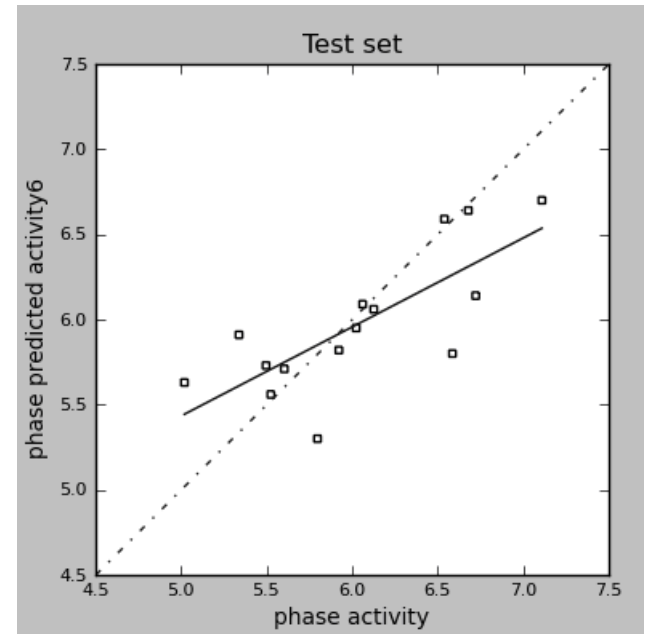

Fig 4: Scatter plots for the predicted and experimental pIC50 values for the ADAMTS-5 QSAR model applied to the test set.

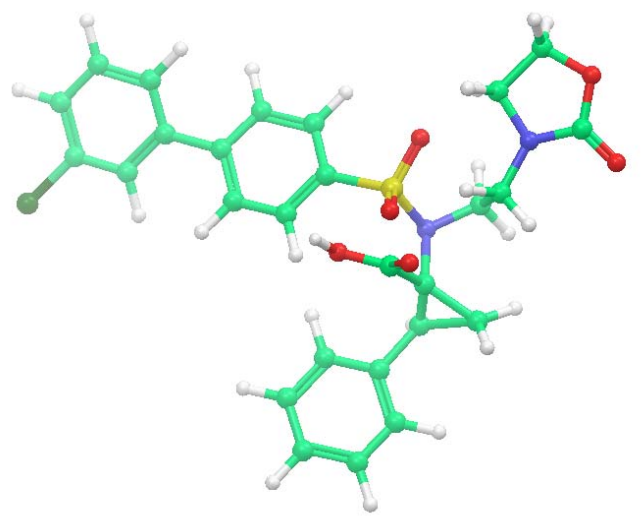

Fig: 5 Compound 10: sulfonylamino-alkanecarboxylate, 38

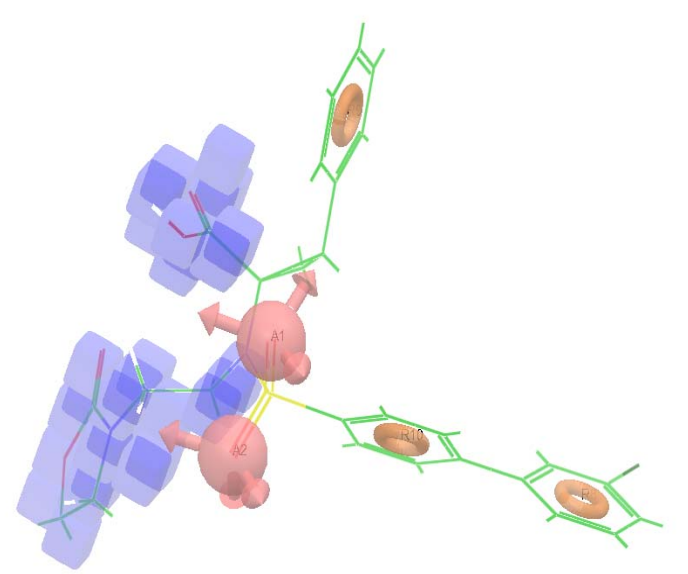

Fig 6: Atom based 3D QSAR model visualized in the context of most active compound 10. (Blue cubes indicate favorable regions while red cubes indicate unfavorable region for the activity) 


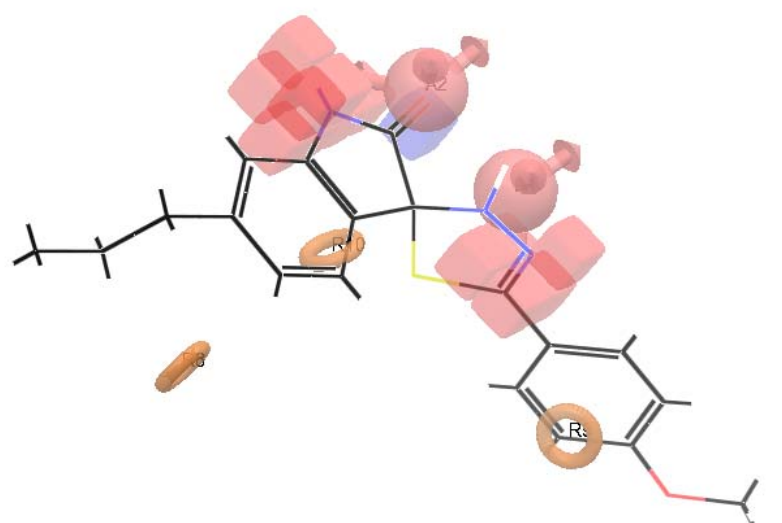

Fig 7: Atom based 3D QSAR model visualized in the context of least active compound 20 .

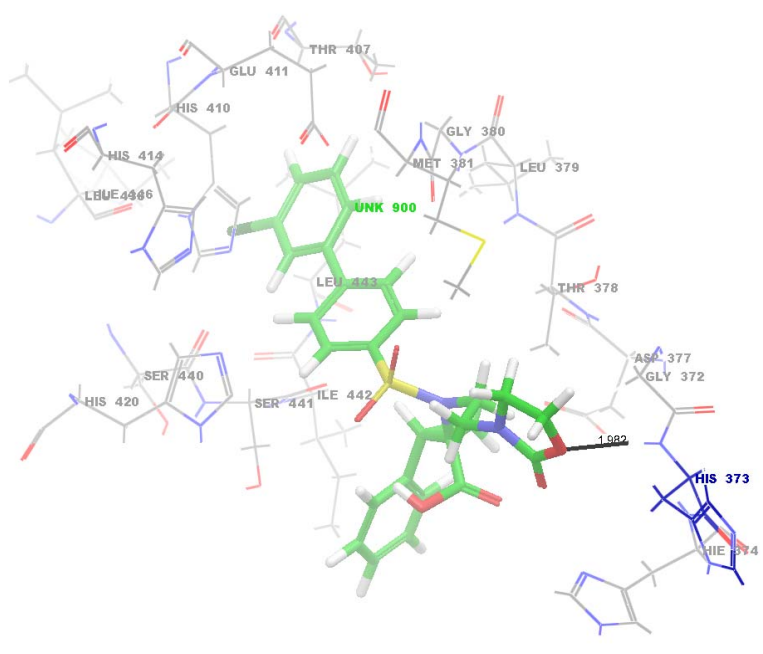

Fig 8: Docking of compound 10 in the active site of ADAMTS-5

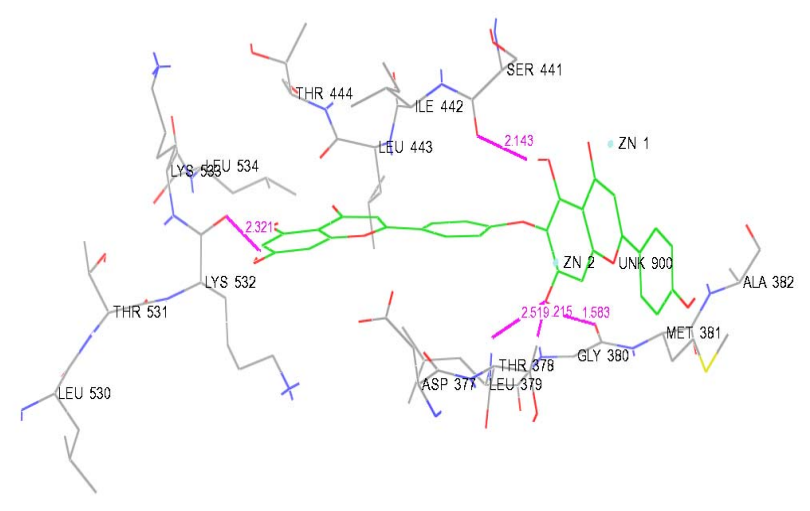

Fig: 9 Docking with natural compound Hinokiflavone

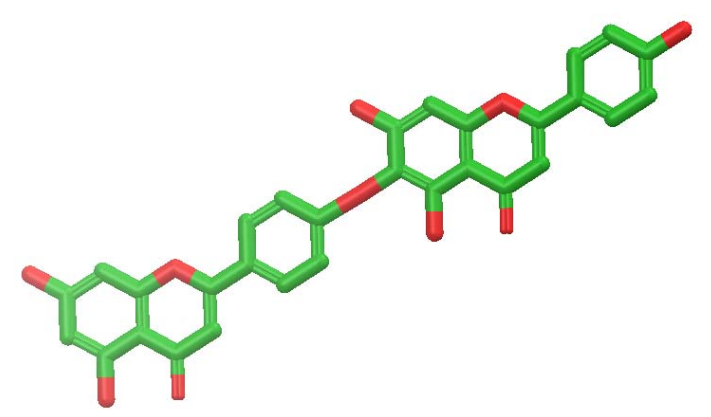

Fig: 10 Structure of Hinokiflavone

TABLE I. BEST 5 HYPOTHESES GENERATED

\begin{tabular}{|c|c|c|c|}
\hline S. No & ID & Survival score & $\begin{array}{c}\text { Survival inactive } \\
\text { score }\end{array}$ \\
\hline 1 & AARRR.4144 & 3.821 & 2.296 \\
\hline 2 & AARRR.3936 & 3.798 & 1.987 \\
\hline 3 & AARRR.4159 & 3.774 & 2.282 \\
\hline 4 & AARRR.4283 & 3.749 & 1.953 \\
\hline 5 & AHRRR.2859 & 3.727 & 2.394 \\
\hline
\end{tabular}

TABLE II. RESULTS OF ATOM-BASED 3D-QSAR WITH PLS 6 AARRR.4144 HYPOTHESIS.

\begin{tabular}{|c|l|l|l|l|l|l|}
\hline ID & $\#$ & SD & $\mathrm{R}^{2}$ & \multicolumn{1}{|c|}{ RMSE } & $\mathrm{Q}^{2}$ & Pearson-R \\
\hline \multirow{2}{*}{ AARRR. } & 1 & 0.38 & 0.604 & 0.427 & 0.4455 & 0.6676 \\
4144 & 2 & 0.2 & 0.887 & 0.4 & 0.5124 & 0.7286 \\
& 3 & 0.15 & 0.942 & 0.372 & 0.5789 & 0.7742 \\
& 4 & 0.13 & 0.96 & 0.369 & 0.5851 & 0.7783 \\
& 5 & 0.08 & 0.983 & 0.383 & 0.5532 & 0.756 \\
& 6 & 0.07 & 0.989 & 0.38 & 0.561 & 0.7593 \\
\hline
\end{tabular}

$\mathrm{SD}=$ standard deviation of the regression, $\mathrm{R}^{2}=$ correlation coefficient, $\mathrm{Q}^{2}=$ for the predicted activities, RMSE $=$ root-mean-square error, Pearson- $\mathrm{R}=$ correlation between the predicted and observed activity for the test set

TABLE III. THE BEST FIVE COMPOUNDS FOR AARRR.4144 HYPOTHESIS.

\begin{tabular}{|l|l|l|l|l|l|}
\hline S. No & $\begin{array}{c}\text { Compo } \\
\text { und \# }\end{array}$ & $\begin{array}{c}\text { QSAR } \\
\text { Set }\end{array}$ & $\begin{array}{c}\text { Experi } \\
\text { mental } \\
\text { pIC50 }\end{array}$ & $\begin{array}{c}\text { Predicted } \\
\text { pIC50 }\end{array}$ & Fitness \\
\hline 1 & 10 & training & 6.658 & 6.82 & 3 \\
\hline 2 & 5 & training & 6.745 & 6.79 & 2.97 \\
\hline 3 & 13 & training & 6.569 & 6.75 & 2.97 \\
\hline 4 & 9 & test & 6.678 & 6.78 & 2.95 \\
\hline 5 & 12 & training & 6.569 & 6.76 & 2.95 \\
\hline
\end{tabular}

TABLE IV. THE DISTANCE BETWEEN THE DIFFERENT SITES OF AARRR.4144 HYPOTHESIS

\begin{tabular}{|r|c|r|}
\hline Site1 & Site2 & Distance \\
\hline A1 & A2 & 2.553 \\
\hline A1 & R8 & 8.089 \\
\hline A1 & R9 & 4.999 \\
\hline A1 & R10 & 3.908 \\
\hline A2 & R8 & 8.122 \\
\hline A2 & R9 & 7.171 \\
\hline A2 & R10 & 3.918 \\
\hline R8 & R9 & 9.248 \\
\hline R8 & R10 & 4.33 \\
\hline R9 & R10 & 6.461 \\
\hline
\end{tabular}


TABLE V. THE ANGLES BETWEEN THE DIFFERENT SITES OF AARRR.4144 HYPOTHESIS

\begin{tabular}{|c|c|c|c|}
\hline Site 1 & Site2 & Site3 & Angle \\
\hline $\mathrm{A} 2$ & $\mathrm{~A} 1$ & $\mathrm{R} 8$ & 81.7 \\
\hline $\mathrm{A} 2$ & $\mathrm{~A} 1$ & R9 & 141.3 \\
\hline $\mathrm{A} 2$ & $\mathrm{~A} 1$ & $\mathrm{R} 10$ & 71.2 \\
\hline $\mathrm{R} 8$ & $\mathrm{~A} 1$ & R9 & 86.5 \\
\hline $\mathrm{R} 8$ & $\mathrm{~A} 1$ & $\mathrm{R} 10$ & 11.5 \\
\hline R9 & $\mathrm{A} 1$ & $\mathrm{R} 10$ & 92.2 \\
\hline $\mathrm{A} 1$ & $\mathrm{~A} 2$ & $\mathrm{R} 8$ & 80.2 \\
\hline $\mathrm{A} 1$ & $\mathrm{~A} 2$ & R9 & 25.8 \\
\hline $\mathrm{A} 1$ & $\mathrm{~A} 2$ & $\mathrm{R} 10$ & 70.7 \\
\hline $\mathrm{R} 8$ & $\mathrm{~A} 2$ & R9 & 74.1 \\
\hline $\mathrm{R} 8$ & $\mathrm{~A} 2$ & $\mathrm{R} 10$ & 10.6 \\
\hline R9 & $\mathrm{A} 2$ & $\mathrm{R} 10$ & 63.6 \\
\hline $\mathrm{A} 1$ & $\mathrm{R} 8$ & A2 & 18.1 \\
\hline $\mathrm{A} 1$ & $\mathrm{R} 8$ & R9 & 32.7 \\
\hline $\mathrm{A} 1$ & $\mathrm{R} 8$ & $\mathrm{R} 10$ & 10.4 \\
\hline $\mathrm{A} 2$ & $\mathrm{R} 8$ & R9 & 48.2 \\
\hline $\mathrm{A} 2$ & $\mathrm{R} 8$ & $\mathrm{R} 10$ & 9.6 \\
\hline R9 & R8 & $\mathrm{R} 10$ & 38.7 \\
\hline $\mathrm{A} 1$ & R9 & A2 & 12.9 \\
\hline $\mathrm{A} 1$ & R9 & $\mathrm{R} 8$ & 60.8 \\
\hline $\mathrm{A} 1$ & R9 & $\mathrm{R} 10$ & 37.2 \\
\hline $\mathrm{A} 2$ & R9 & $\mathrm{R} 8$ & 57.6 \\
\hline $\mathrm{A} 2$ & R9 & $\mathrm{R} 10$ & 32.9 \\
\hline R8 & R9 & $\mathrm{R} 10$ & 24.8 \\
\hline $\mathrm{A} 1$ & $\mathrm{R} 10$ & A2 & 38.1 \\
\hline $\mathrm{A} 1$ & $\mathrm{R} 10$ & $\mathrm{R} 8$ & 158.2 \\
\hline $\mathrm{A} 1$ & $\mathrm{R} 10$ & R9 & 50.6 \\
\hline $\mathrm{A} 2$ & $\mathrm{R} 10$ & $\mathrm{R} 8$ & 159.9 \\
\hline $\mathrm{A} 2$ & $\mathrm{R} 10$ & R9 & 83.6 \\
\hline $\mathrm{R} 8$ & $\mathrm{R} 10$ & R9 & 116.6 \\
\hline
\end{tabular}

\section{ACKNOWLEDGMENT}

Authors thank SRM University for their constant support and their funding for carrying out this study.

\section{REFERENCES}

[1] Wolfsberg TG, Primakoff P, Myles DG, White JM, "ADAM, a novel family of membrane proteins containing A Disintegrin And Metalloprotease domain: multipotential functions in cell-cell and cell-matrix interactions.”, J Cell Biol. 1995 Oct;131(2):275-8.

[2] Seals DF, Courtneidge SA, "The ADAMs family of metalloproteases: multidomain proteins with multiple functions." Genes Dev. 2003 Jan $1 ; 17(1): 7-30$
[3] Fosang AJ, Rogerson FM, East CJ, Stanton H, "ADAMTS-5: the story so far”, Eur Cell Mater. 2008 Feb 5;15:11-26.

[4] Cudic M, Burstein GD, Fields GB, Lauer-Fields J., " Analysis of flavonoid-based pharmacophores that inhibit aggrecanases (ADAMTS-4 and ADAMTS-5) and matrix metalloproteinases through the use of topologically constrained peptide substrates". Chem Biol Drug Des. 2009 Nov;74(5):473-82.

[5] http://www.bindingdb.org/bind/index.jsp

[6] Ujashkumar A. Shah, Hemantkumar S. Deokar, Shivajirao S. Kadam and Vithal M. Kulkarni, "Pharmacophore generation and atom-based 3D-QSAR of novel 2-(4-methylsulfonylphenyl)pyrimidines as COX2 inhibitors.", 2010 Aug;14(3):559-568.

[7] Tawari NR, Bag S, Degani MS, "Pharmacophore mapping of a series of pyrrolopyrimidines, indolopyrimidines and their congeners as multidrug-resistance-associated protein (MRP1) modulators.", J Mol Model. 2008 Oct;14(10):911-21.

[8] Vipin Kumar*, Sunil Kumar and Poonam Rani, "Pharmacophore modeling and 3D-QSAR studies on flavonoids as $\alpha$ - glucosidase inhibitors.", Der Pharma Chemica, 2010, 2(4): 324-335.

[9] Steven L. Dixon, Alexander M. Smondyrev and Shashidhar N. Rao, "PHASE: A Novel Approach to Pharmacophore Modeling and 3D Database Searching,", Chem Biol Drug Des 2006; 67: 370-372.

[10] Dixon SL, Smondyrev AM, Knoll EH, Rao SN, Shaw DE, Friesner RA, "PHASE: a new engine for pharmacophore perception, 3D QSAR model development, and 3D database screening: 1. Methodology and preliminary results", J Comput Aided Mol Des (2006) 20:647-671.

[11] Prashant Revan Murumkar, Vishal Prakash Zambre and Mange Ram Yadav, "Development of predictive pharmacophore model for in silico screening, and 3D QSAR CoMFA and CoMSIA studies for lead optimization, for designing of potent tumor necrosis factor alpha converting enzyme inhibitors", Comput Aided Mol Des (2010) 24:143-156.

[12] Darren F. Seals and Sara A, "The ADAMs family of metalloproteases: multidomain proteins with multiple functions.", Genes Dev 2003 17: 7-30 .

[13] Manas K. Majumdar, Roger Askew, et al, "Double-Knockout of ADAMTS-4 and ADAMTS-5 in Mice Results in Physiologically Normal Animals and Prevents the Progression of Osteoarthritis", November 2007, ARTHRITIS \& RHEUMATISM, Vol. 56, No. 11, pp 3670-3674.

[14] Matthew G. Bursavich,a, Adam M. Gilbert, et al. "Synthesis and evaluation of aryl thioxothiazolidinone inhibitors of ADAMTS-5 (Aggrecanase-2).”, (2007), Bioorganic \& Medicinal Chemistry Letters 17 1185-1188.

[15] http://www.pdb.org/pdb/home/home.do

[16] http://www.ars-grin.gov/duke/

[17] Plaas A, Li J, Riesco J, Das R, Sandy JD, Harrison A. .Intra-articular injection of hyaluronan prevents cartilage erosion, periarticular fibrosis and mechanical allodynia and normalizes stance time in murine knee osteoarthritis. Arthritis Res Ther. 2011 Mar 20;13(2):R46.

[18] Plaas A, Sandy JD, Liu H, Diaz MA, Schenkman D, Magnus RP, Bolam-Bretl C, Kopesky PW, Wang VM, Galante JO. Biochemical identification and immunolocalizaton of aggrecan, ADAMTS5 and inter-alpha-trypsin-inhibitor in equine degenerative suspensory ligament desmitis. J Orthop Res. 2011 Jan 18. doi: 10.1002/jor.21332.

[19] Durigova M, Troeberg L, Nagase H, Roughley PJ, Mort JS. Involvement of ADAMTS5 and hyaluronidase in aggrecan degradation and release from OSM-stimulated cartilage. Eur Cell Mater. 2011 Jan 12;21:31-45.

[20] Durigova M, Nagase H, Mort JS, Roughley PJ. MMPs are less efficient than ADAMTS5 in cleaving aggrecan core protein. Matrix Biol.2011Mar;30(2):145-53. 